This item was submitted to Loughborough's Research Repository by the author.

Items in Figshare are protected by copyright, with all rights reserved, unless otherwise indicated.

\title{
Test and evaluation of a fuzzy diagnostic model for PEFC
}

\section{PLEASE CITE THE PUBLISHED VERSION}

https://www.crcpress.com/Risk-Reliability-and-Safety-Innovating-Theory-and-Practice-Proceedings/WallsRevie-Bedford/p/book/9781138029972

\section{PUBLISHER}

(c) Taylor \& Francis

\section{VERSION}

AM (Accepted Manuscript)

\section{PUBLISHER STATEMENT}

This work is made available according to the conditions of the Creative Commons Attribution-NonCommercialNoDerivatives 4.0 International (CC BY-NC-ND 4.0) licence. Full details of this licence are available at: https://creativecommons.org/licenses/by-nc-nd/4.0/

\section{LICENCE}

CC BY-NC-ND 4.0

\section{REPOSITORY RECORD}

Davies, Benjamin, Lisa M. Jackson, and Sarah J. Dunnett. 2017. "Test and Evaluation of a Fuzzy Diagnostic Model for PEFC". figshare. https://hdl.handle.net/2134/24574. 


\title{
Test and Evaluation of a Fuzzy Diagnostic Model for PEFC
}

\author{
B. Davies, L. Jackson, S. Dunnett \\ Department of Aeronautical and Automotive Engineering, Loughborough University, United Kingdom
}

\begin{abstract}
Diagnosing faulty conditions of engineering systems is a highly desirable process within control structures, such that condition based maintenance (CBM) is made possible and effective. The goal herein is to enhance lifetime performance and extend system availability. Difficulty arises in developing a mathematical model which can describe all working and failure modes of complex systems. However the human ability to diagnose a system through experience is a powerful one, and can be represented in fuzzy logic. This paper presents the knowledge base of degradation modes in a polymer electrolyte fuel cell (PEFC), captured from experts and experimental findings. The defined rules produced for the fuzzy logic model connect observed operational modes and symptoms to component degradation. The system is then tested against common automotive stress conditions to assess operability.
\end{abstract}

\section{INTRODUCTION}

Polymer Electrolyte Fuel Cells (PEFCs) have many opportunities as power sources within a variety of markets, ranging from handheld portable electronics to large stationary combined heat and power systems (Sharaf \& Orhan 2014; Piela \& Mitzel 2015). One of the largest opportunities is the transport sector, where government and industry are already working towards decarbonisation in the face of climate change (Prins et al. 2010).

However, PEFC systems are yet to reach full commercialisation, largely due to major hurdles in fuel infrastructure, system cost, and lifetime durability. Different aspects of research and industry are working to overcome these drawbacks. New infrastructure for charging battery electric vehicles (BEVs) has shown a working framework, which could similarly be applied in the deployment of hydrogen refuelling stations (Thomas 2009). To be technologically competitive PEFC system costs need to be comparable to traditional power plant technologies, i.e. $30 \$ \mathrm{~kW}^{-1}$ (Transactions \& Society 2007). This is largely a materials approach, including Ptloading and carbon nano-structures, and is likely to decrease with further development and manufacturing effort.

The durability of PEFC systems under automotive operating conditions remains a working objective. Fuel cell vehicles are required to perform and survive comparably to internal combustion powertrains; nominally 5000 hrs total lifetime, or 150,000 mi equivalent usage, without a $10 \%$ drop-off in output (Garland et al. 2012).

Automotive applications for PEFC systems are expected to introduce a wide range of operating conditions. Rapid start-up and load cycling is imposed freely in automotive driving, as well as various environmental conditions $\left(-40\right.$ to $+40{ }^{\circ} \mathrm{C}$ for example). Unfortunately, at present, the best demonstration projects are only reporting up to 2500 hrs, showing room for significant improvement (Wipke et al. 2012).

Prognostics and Health Management (PHM) offers a solution to this issue. PHM is a set of techniques to assess performance, diagnose faults, predict remaining useful life (RUL), and make control decisions to enhance lifetime durability. This project aims to develop a PHM system for PEFC systems, through the diagnosis-oriented model presented herein.

Output performance of a PEFC is quantified in the voltage generated, and can be modelled as a function of the current drawn by the external load as given in equation 1(Jouin et al. 2016):

$$
\begin{aligned}
E= & E_{\text {rev }}-\frac{R T}{n \alpha F} \cdot \ln \left(\frac{i+i_{\text {loss }}}{i_{0}}\right)-i \cdot r_{\text {cell }} \\
& +B_{C} \cdot \ln \left(1-\frac{i}{i_{\max }}\right)
\end{aligned}
$$

where $E$ is the cell voltage, $E_{\text {rev }}$ is the theoretical reversible voltage, $R$ the universal gas constant, $T$ the 
temperature, $n$ the reaction charge number, $\alpha$ the charge transfer coefficient, $F$ the Faraday constant, $i$ the current density, $i_{\text {loss }}$ the hydrogen crossover current, $i_{0}$ the reaction exchange current, $r_{\text {cell }}$ the internal cell resistance, $B_{C}$ is an empirical parameter taking into account water and gas accumulation, and $i_{\max }$ the limiting cathode current.

This expression gives the output of the PEFC under steady state conditions (temperature, pressure, concentration, etc.) and does not take into account any losses due to damage. It is difficult to apply this model in dynamic loading situations, as in automotive power, because of the inability to accurately measure internal concentrations and current densities in this situation.

Mathematical modelling is made all the more complex when considering degradation behaviour of the fuel cell also. Damage to the polymer electrolyte membrane, Pt-catalyst particles, or carbon diffusion electrodes will decrease voltage output throughout the PEFC's lifetime. Thus, a fuzzy description of fuel cell degradation behaviour has been conceived.

This paper details the developments made to a fuzzy diagnostic model of the PEFC. This calls for an understanding of the operational conditions causing component degradation. In the second part of the paper the experimental set up is discussed, along with initial validation results.

\section{DIAGNOSIS}

The goal of the diagnostic process is to inform the operator and control system when a fault is occurring, and how severe the damage is to ongoing system output. This information will allow said parties to assess system health, and decide on alterations to future usage.

As in most health management methods, the first process is to compare observed system variables to the reference values expected for the same operating conditions. Whilst fuel cell design and experimental validation provides a suggested operation point in terms of temperature, stoichiometry, electronic load, among others, functional use will exert the PEFC to many off-design conditions. The resulting difference is a residual between optimal and potentially faulty operation.

The calculated residual gives an indication that a fault of some nature is occurring; a detection that the PEFC is performing in off design conditions. This may be sufficient for control systems to alter inputs and realign with designed use, or to alert the user that a fault has occurred.

The next stage process is to diagnose the cause of the reduced performance. Commonly this is calculated through mathematical models, when the system process is well known. In the case of PEFC electrochemistry, the system processes are more complex, and some parameters are not measurable outside lab conditions. This is where fuzzy logic provides the solution to the diagnostic problem.

Fuzzy modelling applies expert knowledge to the PEFC system; where experimental observations hold more information than can be successfully expressed mathematically. The fuzzy model is a system of IFTHEN statements which describe the relationship between observable external variables and the inferred internal workings of the fuel cell. This does require understanding of the degradation processes, as described in the following section.

\subsection{PEFC degradation}

Among the many components of the PEFC, the most important to safe and efficient operation is the membrane electrode assembly (MEA), shown in figure 1. The MEA is the central part of the fuel cell reaction; it governs the transport of reactants, defines the internal cell resistance, and supports the catalytic reaction sites. The MEA is also the most expensive component in the construction, so management of this component's durability will have lifetime cost implications.

The MEA is constructed of the polymer electrolyte membrane, Nafion being the most popularly employed in PEFCs, the platinum catalyst nanoparticles, supported on carbon micro-structures, and a gas diffusion electrode (GDE) backing, commonly a carbon-based cloth. Each of these materials has different mechanisms and conditions for degradation to occur.

The Nafion membrane is most susceptible to mechanical and chemical breakdowns of the polymer structure. Mechanical stresses arise from the physical clamping within the fuel cell construction, and can be accelerated as the membrane changes size

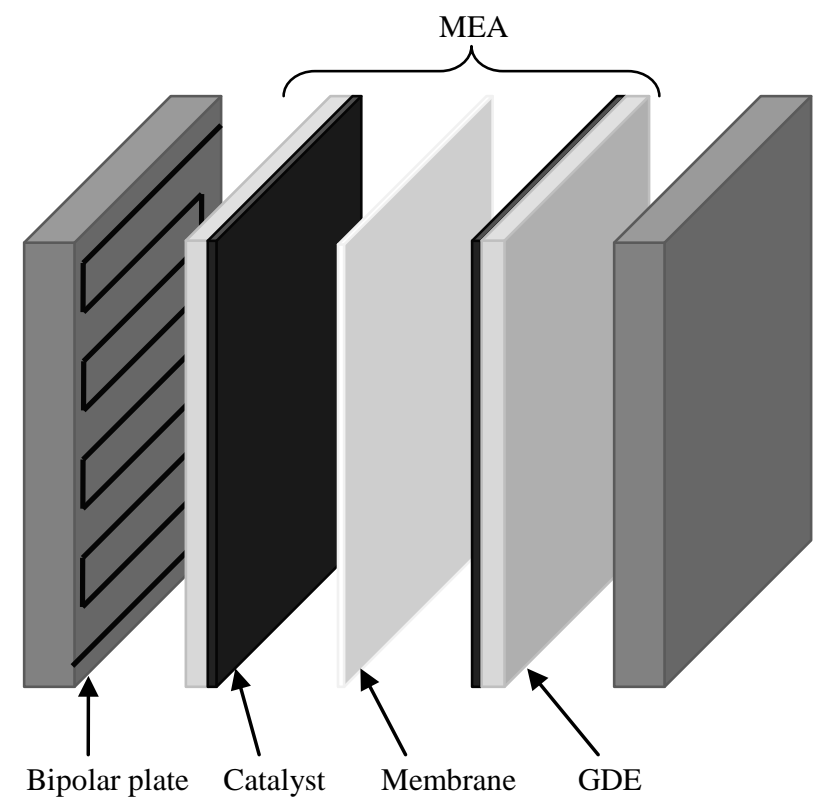

Figure 1: Components of the PEFC stack 
with water absorption. Chemical attacks come from hydroxide radical attacks, notably arising from open circuit voltage (OCV) operation.

The platinum catalyst is distributed as nanoparticles to maximise surface area, however they are known to agglomerate to decrease their surface energy state. This has the secondary effect of reducing the surface area available for reaction. Catalyst reaction area can also be reduced by surface oxide formation. Both these degradation modes are caused by excess humidity, and operation at cyclical, high or open circuit voltages.

The carbon materials in the PEFC are largely associated with diffusion processes. This means the pores within the GDE can become blocked by liquid water, despite hydrophobic treatment, especially during high humidity operation. The carbon materials can also be directly consumed by the anode reaction during fuel starvation conditions.

Water management is also vitally important to the PEFC reaction; the polymer membrane must be fully hydrated for optimal charge transfer. This means water is often introduced to the fuel cell in the gas supply, as well as being produced by the hydrogenoxygen reaction itself. This does however mean that an excess of liquid water in the gas diffusion materials can occur, blocking pathways to reaction sites. Water balance is a function of the amount of water introduced in humidification and as a reaction product, as well as the cell temperature.

\subsection{Fuzzy diagnostic model}

The previous section describes the most pertinent degradation modes and their causes. This is a qualitative analysis, and this is where the fuzzy logic functions. The rules system is able to take the linguistic description of variables and degradation severity, and apply these to the measured PEFC system.

Table 1 gives the rules base for the PEFC diagnosis. This gives an indication of the likelihood of any one degradation mode being the cause of performance drop off. These rules use a small set of inputs, meaning fewer sensor measurements required. Cell temperature and voltage are relatively simple to measure, whilst gas humidity is the most expensive

Table 1: Simplified diagnostic rules base

\begin{tabular}{ll}
\hline IF & THEN \\
\hline Open circuit operation & Membrane chemical breakdown \\
Humidity cycling quickly & Membrane mechanical stress \\
Voltage cycling (range & Catalyst dissolution increase \\
0.7 to 1.2 V) & \\
Fuel supply decreases & Carbon support corrosion \\
Temperature low OR & Flooding event rate increases \\
feed humidity increases & \\
OR current load high & \\
Temperature high OR & Membrane dehydration increases \\
feed humidity decreases & \\
\hline
\end{tabular}

sensor requirement. This should keep the overall cost of the diagnostic package low, so as not to inflate the PEFC system cost.

In addition to selecting which degradation mode is in action, the diagnostic model makes a severity assessment. This is through comparison to the steadystate equation 1 as a residual against a reference voltage performance. Though the steady-state equation was said to be too simplistic for full diagnosis, it is this simplicity which allows quick computation of an expected output.

Severity is also modelled using fuzzy logic; purely based on voltage performance. Table 2 gives these severity thresholds, diverging from the reference value, and in keeping with the DoE lifetime durability target of less than $10 \%$ loss.

\section{EXPERIMENTAL}

\subsection{Test rig}

All tests were carried out on a $100 \mathrm{~cm}^{2}$ Pragma PEFC, single cell, which includes 7-fold serpentine gas flowfields. Gas supplies are air at the cathode and hydrogen at the anode, with a nitrogen purge for start-up/shut-down protocols. In addition to the gas feeds, the fuel cell has an internal water circuit for heat management.

Tests were carried out using purpose designed control and monitoring software. A standard procedure has been followed for cell start-up and conditioning (Piela \& Mitzel 2015).

\subsection{Water management}

The chosen method, for inducing a degradation state and the diagnosis thereof, was to vary stack temperature under constant loading conditions. This has the effect of changing water balance in the cell, creating either flooding or dehydration states. Thermal cycling is thought to be representative of the environmental conditions expected in automotive usage.

Base performance was established with steady state conditions; stack temperature at $60{ }^{\circ} \mathrm{C}$, both hydrogen and air supplies humidified to $30{ }^{\circ} \mathrm{C}$ dew point, and cell voltage at $0.65 \mathrm{~V}$ (20 A loading). This humidity level is relatively low for optimal performance, though better suited to the temperature ranges for the following procedures.

Table 2: Degradation severity rules base

\begin{tabular}{ll}
\hline IF & THEN \\
\hline Voltage drop is $0-1 \%$ & Non-severe \\
Voltage drop is $1-3 \%$ & Low severity \\
Voltage drop is $3-5 \%$ & Medium severity \\
Voltage drop is $5-10 \%$ & High severity \\
Voltage drop is $10+\%$ & Severity warning \\
\hline
\end{tabular}



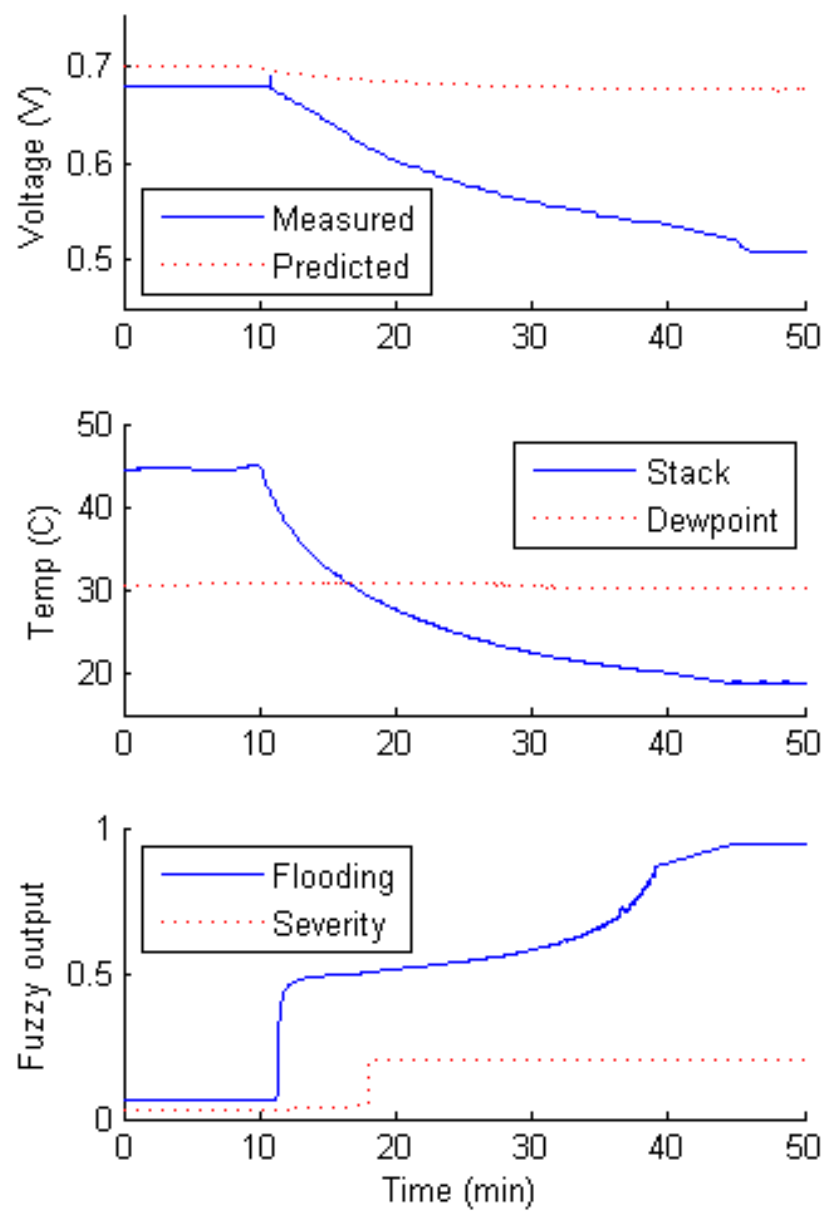

Figure 2: Diagnostic results for flooding test

Flooding tests were initiated by reducing stack temperature. This was controlled by reducing the temperature of the coolant water circuit. After a few minutes the stack temperature would drop to $20{ }^{\circ} \mathrm{C}$. At the lower temperature, water is more likely to condense within the flow fields and GDE, blocking the gas diffusion, and inducing mass transport losses. Depending on the scale of the flooding event, this may be a purely localised gas starvation, or more homogenously across the active area; this distinction is not yet possible in the diagnostic system.

Dehydration states were achieved through the reverse process; increasing the stack temperature beyond the dew point temperature. This implies the PEFC is operating in negative water balance, losing more in the exhaust than is being introduced or created.

\section{RESULTS AND DISCUSSION}

\subsection{Flooding event}

The first fault experimentally diagnosed is for flooding events; the accumulation of liquid water in the gas diffusion media. Figure 2 presents the experimental results obtained for the test; cell temperature
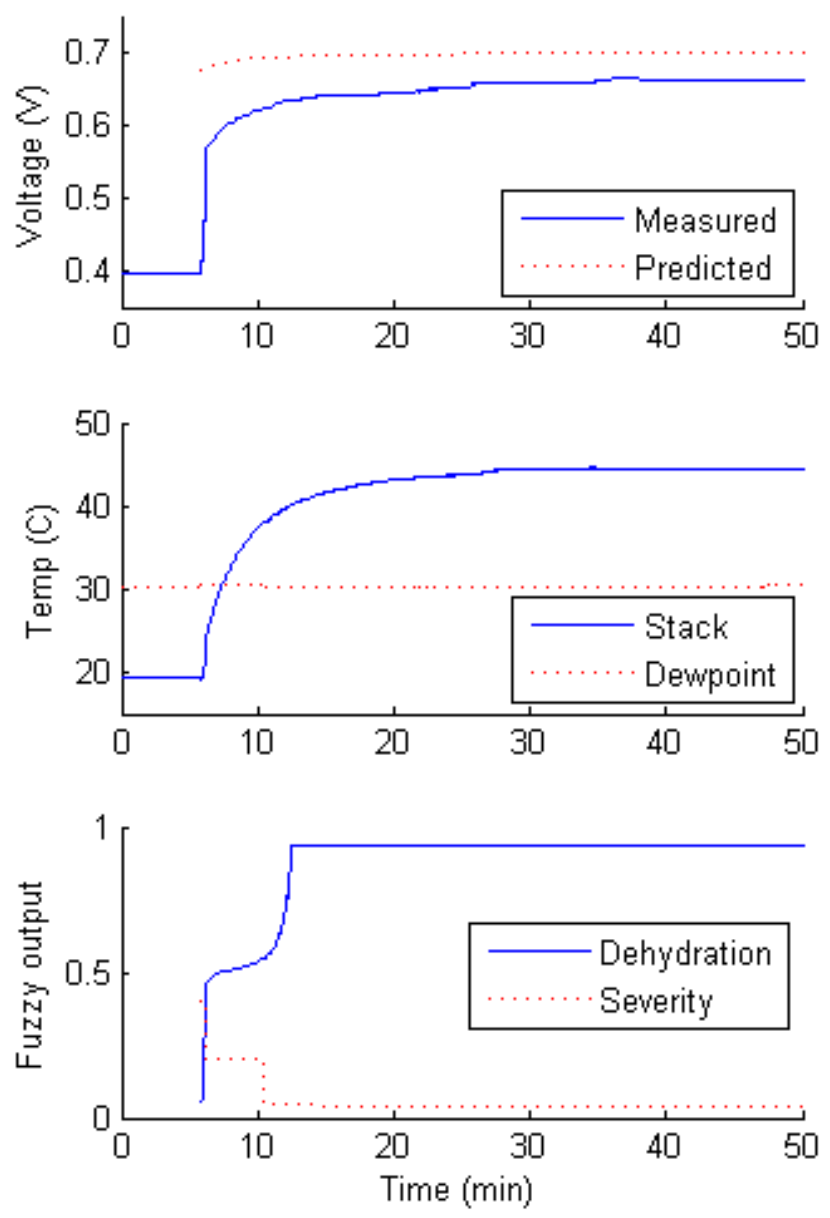

Figure 3: Diagnostic results for dehydration test

is reduced as time progresses.

At the beginning of the experiment, with temperature and current loading held constant, voltage output was stable. The fuzzy logic response for flooding was therefore constant and low (a small positive response is seen thanks to stack temperature being bellow the design value of $60{ }^{\circ} \mathrm{C}$ ). Together with is the small variance between measured and reference voltage gives a low severity degradation state.

The line representing the flooding diagnosis shows good response to the temperature change. Predicted likelihood of flooding events increases as temperature decreases. The response ramps up quickly, approaching the defined dew point temperature, and again as temperature drops even further. By the end of the test, flooding is almost certainly predicted by the diagnosis.

Voltage output is seen to decrease with temperature change. This is expected; temperature is fundamental to the PEFC reaction mechanisms, as seen in equation 1 . The voltage does however diverge from the reference value, calculated by this equation implying addition mass transport losses due to liquid water presence.

Severity response increases from the none- to the low severity case as the measured voltage decreases. 
The result is informing the operator that flooding events are likely to be occurring in the fuel cell, with minimal impact to the performance; action should be taken to increase stack temperature, before flooding becomes more damaging to the PEFC.

\subsection{Dehydration}

The second experiment is for membrane dehydration. Figure 3 presents the time progression for the test, as cell temperature is increased beyond the gas supply dew point.

Under this test, the fuzzy logic threshold for dehydration conditions was set purposefully low, compared to the design operation temperature. One limitation of the test environment is in how much heat the PEFC is able to generate. Nonetheless, increasing the stack temperature beyond dew point will induce low humidity conditions.

As with the flooding response previously, the fuzzy diagnostic response is quick to acknowledge the high temperature conditions. This infers the capacity for membrane dehydration. Membrane dehydration does however take several minutes of consistently hot and dry operation to cause noticeable effect to the fuel cell conductivity, so the diagnosis should be combined with the severity monitor.

Stack voltage increases with temperature, in keeping with the analysis of equation 1 . There is however no divergence from the reference voltage during the progression of this test. Dehydration of the membrane will take longer to manifest as output degradation, and so this is not seen in the voltage measurement. In further testing, the dehydration conditions should be maintained for longer to allow more severe degradation, and a resolute validation.

\section{CONCLUSIONS}

An approach to diagnosis of common PEFC degradation modes is proposed herein, utilising fuzzy logic. The linguistic rules base to this monitoring system is built on expert understanding of accelerated conditions for common degradation modes. The goal of this diagnostic model is to provide real-time response to degradation phenomena, forgoing traditional discrete tests, and allowing operators and control systems to intelligently maintain PEFC performance.

The fuzzy diagnostic model is validated experimentally on a single cell PEFC test rig. Although this is a smaller, low power PEFC than would be used in automotive applications, the degradation phenomena are notably present in all scales of the technology. Functions of the fuzzy logic diagnosis can be extrapolated to larger power plants, taking in- to account different degradation rates where appropriate.

Experimental tests are run to stimulate water management difficulties, namely flooding and membrane dehydration, a common occurrence in dynamic applications. The fuzzy logic system responded rapidly to changing operational conditions, although the potential rate of degradation could be better modelled for slow evolving modes.

Future work will continue to validate the diagnosis of other degradation modes, as well as tuning the response rate to better reflect PEFC dynamics. Building on this, control responses will be developed to restore optimal conditions, mitigate further degradation damage, and manage dynamic operation.

\section{ACKNOLEDGEMENTS}

This project is supported by the Engineering and Physical Science Research Council (grant number EP/L015749/1).

\section{REFERENCES}

Garland, N.L., Papageorgopoulos, D.C. \& Stanford, J.M., 2012. Hydrogen and Fuel Cell Technology: Progress, Challenges, and Future Directions. Energy Procedia, 28, pp.2-11.

Jouin, M. et al., 2016. Degradations analysis and aging modeling for health assessment and prognostics of PEMFC. Reliability Engineering \& System Safety, 148, pp.78-95.

Piela, P. \& Mitzel, J., 2015. Polymer electrolyte membrane fuel cell efficiency at the stack level. Journal of Power Sources, 292, pp.95-103.

Prins, G. et al., 2010. The Hartwell Paper.

Sharaf, O.Z. \& Orhan, M.F., 2014. An overview of fuel cell technology: Fundamentals and applications. Renewable and Sustainable Energy Reviews, 32, pp.810-853.

Thomas, C.E., 2009. Fuel cell and battery electric vehicles compared. International Journal of Hydrogen Energy, 34, pp.6005-6020.

Transactions, E.C.S. \& Society, T.E., 2007. DOE Fuel Cell Program: Durability Technical Targets and Testing Protocols N. L. Garland*. ECS Transactions, 11(1), pp.923-931. 
Wipke, K. et al., 2012. National Fuel Cell Electric Vehicle Learning Demonstration Final Report National Fuel Cell Electric Vehicle Learning Demonstration Final Report. NREL / DoE, (July). 